\title{
Ethanol extract of Artemisia sieversiana exhibits anticancer effects and induces apoptosis through a mitochondrial pathway involving DNA damage in COLO-205 colon carcinoma cells
}

\author{
Jun Tang1, Juan-Juan Zhao² and Zhi-Hong Li ${ }^{1}$ \\ ${ }^{1}$ Second Department of General Surgery, Ezhou Central Hospital, Erzhou 436 000, China; ${ }^{2}$ Department of \\ Pathology, Ezhou Central Hospital, Ezhou 436 000, China.
}

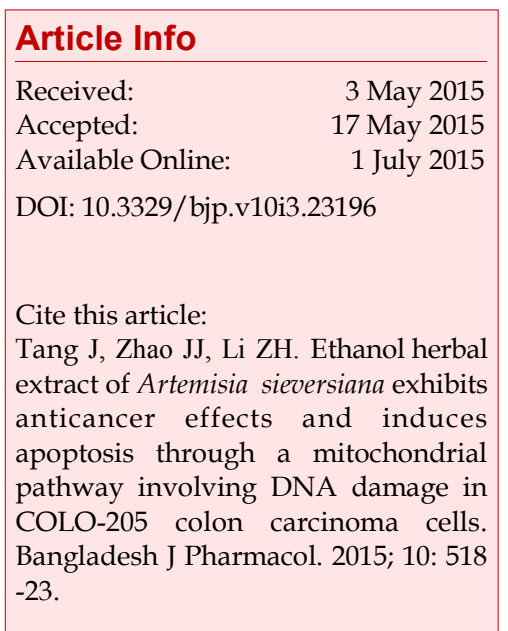

\begin{abstract}
The aim of the study was to see the antiproliferative and apoptotic effects of ethanolic herbal extract of Artemisia sieversiana against three human colon cancer (HT-29, HCT-15 and COLO-205) cells. The cytotoxicity of the extract on these cell lines was evaluated by MTT assay. Phase contrast and fluorescence microscopy using acridine orange/ethidium bromide (AO/ETBR) staining was employed to investigate morphological alterations in COLO-205 cells by the herbal extract. Flow cytometry instrument measured the changes in mitochondrial membrane potential loss while as gel electrophoresis measured DNA damage in these cells. The extract at increasing doses exhibited a strong cytotoxic effect in a dose-dependent manner against all the three colon cancer cell lines. The $\mathrm{IC}_{50}$ values of the extract against HT-29, HCT-15 and COLO-205 cancer cells were found to be $52.1,43.2$ and $38.6 \mu \mathrm{g} / \mathrm{mL}$ respectively. Mitochondrial membrane potential loss $(\Delta \Psi \mathrm{m})$ and DNA fragmentation events were also observed following extract treatment at increasing doses.
\end{abstract}

\section{Introduction}

Colorectal cancer is the fourth leading cause of deaths arising due to cancer and the third most common cancer globally. Around one million new cases of colon cancer are diagnosed every year (Jemal et al., 2011). Colorectal cancer is the second most frequently diagnosed and third highly mortal cancer in females, whereas it is the third most commonly diagnosed and fourth most highly mortal cancer in males. Risk factors for colorectal cancer include obesity, a diet low in fruits and vegetables, a diet high in fat, alcohol, or red meat, physical inactivity, and smoking (Rupnarain et al., 2004; Kinzler and Vogelstein, 1996). The transformation of normal colonic epithelial cells into a lethal colorectal carcinoma is a multi-factorial process. Numerous factors like cell proliferation, cancer cell migration, angiogenesis and inflammation play crucial roles in colorectal cancer growth and progression. Both adjuvant and neo-adjuvant chemotherapy approaches are useful for colorectal cancer treatment. Conventional chemotherapy comprising 5-fluorouracil, leucovorin, capecitabine and irinotecan regimens for the treatment of colorectal cancer have insufficient effectiveness and are accompanied with significant life-threatening sideeffects (Mano and Duhoux, 2008; Moertel, 1994). In spite of recent advances in the therapy of metastatic colorectal cancer, the prognosis of the patients and the treatment options remain poor (O'Connor et al., 2011). Hence there is a pressing need for the design and development of newer, safer and cheap anticancer drugs which can be used to curb deadly colorectal carcinoma effectively. Natural products have been investigated for many years as anticancer chemotherapeutic agents, including anti-colorectal cancer agents.

\section{Materials and Methods}

Plant material and extraction procedure: Artemisia siever- 
siana aerial parts were collected during June-July 2014 from Xiaolongmeng National Forest Park (Mentougou District, Beijing, China). Voucher specimens (Voucher specimen number 14-12-AS-02) of the identified species were deposited with the Herbarium (BNU) of the College of Life Sciences, Beijing Normal University. The aerial parts of the plant were thoroughly washed with tap water, shade dried and then chopped into small pieces. Ethanol (90\%) was used for cold extraction which was carried out for 48 hours in an extraction apparatus. The extract was then concentrated under reduced pressure in a rotary evaporator at $45^{\circ} \mathrm{C}$ and was then kept in a refrigerator at $4^{\circ} \mathrm{C}$ prior to use.

Chemicals and reagents: Growth medium RPMI-1640, minimum essential medium (MEM), fetal calf serum (GIBCO), trypsin, penicillin, MTT, streptomycin, DMSO and phosphate buffer saline (Hangzhou Sijiqing Biological Products Co., Ltd, China) were used in this study. MTT kit, acridine orange/ethidium bromide dye, Hoechst dye were obtained from Roche (USA), annexin V-FITC-propidium iodide apoptosis detection kit was purchased from Sigma-Aldrich Corporation (St. Louis, MO, USA). All other chemicals and solvents used were of the highest purity grade.

Cell lines and culture condition: Three colon cancer cell lines viz., HT-29, HCT-15 and COLO 205 (human colon cancer cells) were procured from Shanghai Institute of Cell Resource Center of Life Science (Shanghai, China). The cells were cultured in minimum essential medium (MEM) supplemented with $10 \%$ fetal bovine serum, 100 $\mathrm{U} / \mathrm{mL}$ penicillin and $100 \mu \mathrm{g} / \mathrm{mL}$ streptomycin. The cells were kept at $37^{\circ} \mathrm{C}$ in a humidified atmosphere containing $5 \% \mathrm{CO}_{2}$. The cells were treated with different concentrations of the $A$. sieversiana extract $(0,15,25$, $35,45,75$ and $125 \mu \mathrm{g} / \mathrm{mL})$.

Cell viability testing by MTT assay: The effect of the A. sieversiana extract on the cell viability was evaluated by the MTT assay, which relies on the reduction of a tetrazolium salt by mitochondrial succinate dehydrogenase in living cells. The cells were seeded in a 96-well plate at a density of $2 \times 10^{5}$ cells $/ \mathrm{mL}$, treated with the extract, and incubated for 48 hours. After that MTT stock solution $(50 \mu \mathrm{L}, 2 \mathrm{mg} / \mathrm{mL})$ was added to each well to attain a total reaction volume of $350 \mu \mathrm{L}$. After incubation for 4 hours, the supernatants were removed. The formazan crystals in each well were then dissolved in $100 \mu \mathrm{L}$ dimethyl sulfoxide (DMSO) and the absorbance was measured in an ELISA reader (Thermo Molecular Devices Co., Union City, USA) at $570 \mathrm{~nm}$.

Phase contrast microscopic study of cellular morphology: Phase contrast microscopy was carried out to assess the morphological alterations in the COLO-205 colon cancer cells. The cells incubated for 48 hours and treated with $A$. sieversiana extract at various concentrations $(0$, 25,45 and $75 \mu \mathrm{g} / \mathrm{mL}$ ). Control cells treated with $0.4 \%$
DMSO alone were also incorporated. The morphological changes were detected and the images were captured under an inverted light microscope (Olympus, PA, USA) after 48 hours. The same spot of cells was marked and photographed.

Fluorescence microscopy using acridine orange/ethidium bromide staining (AO/ETBR): Morphological indication of apoptosis was evaluated by means of acridine orange and ethidium bromide staining as described previously (Martin and Leonardo, 1994). After extract treatment at various doses $(0,25,45$ and $75 \mu \mathrm{g} / \mathrm{mL})$, COLO-205 cells in 8-well chamber slides were stained with acridine orange and ethidium bromide $(10 \mu \mathrm{g} / \mathrm{mL}$ each). Cells were examined by fluorescence microscopy (Olympus BX51 microscope, NY, USA) and photogra-phed using SPOT RT software (Olympus). Acridine orange infiltrates through the cells and makes the nuclei green.

Determination of mitochondrial membrane potential $(\Delta \Psi m)$ in COLO-205 colon cancer cells: The human colon cancer cells (COLO-205) were seeded at $2 \times 10^{5}$ cells/well into 6-well plates. After 24 hours incubation, cells were treated with variable doses $(0,25,45$ and $75 \mu \mathrm{g} / \mathrm{mL})$ of $A$. sieversiana extract for 48 hours. Untreated and treated cells were harvested and washed three times with phosphate buffer saline. The cell pellets were then resuspended in $20 \mathrm{~mL}$ of fresh medium containing 5.0 $\mu \mathrm{M}$ rhodamine- 123 and incubated at $37^{\circ} \mathrm{C}$ in a thermostatic bath for $20 \mathrm{~min}$ with slight shaking and then analyzed using FACS Calibur instrument (BD Biosciences, San Jose, CA) equipped with CellQuest 3.3 software in the Fluorescence-activated Cell Sorting (FACS) machine.

DNA Fragmentation Analysis following A. sieversiana extract treatment: For quantification of DNA fragmentation, cellular DNA fragmentation was evaluated by analysis with a DNA fragmentation kit (Roche Diagnostics, Mannheim, Germany) according to the manufacturer's instructions. The COLO-205 cells $\left(2 \times 10^{5}\right.$ cells/ well) were plated in $6-\mathrm{cm}$ dish and then subjected to the treatment of increasing concentrations $(0,25,45$ and 75 $\mu \mathrm{g} / \mathrm{mL}$ ) of the A. sieversiana extract for 48 hours. After treatment, the cells were washed twice with ice-cold phosphate buffer saline and resuspended in lysis buffer (25 mM Tris- $\mathrm{HCl}, \mathrm{pH} 7.4,5 \mathrm{mM}$ EDTA and 0.5\% SDS) with $3.0 \mathrm{mg} / \mathrm{mL}$ RNase A for $20 \mathrm{~min}$ at $55^{\circ} \mathrm{C}$. Then proteinase $\mathrm{K}$ was added and the cells were incubated overnight. Separation of DNA was performed using $4 \%$ agarose gel and analyzed under UV light after staining with ethidium bromide (ETBR).

Statistical analysis: The values were shown as mean \pm standard error of the mean. Results were analyzed using analysis of variance and Tukey's test to determine pair wise differences. $\mathrm{P}<0.05$ was considered significant. 


\section{Results}

A. sieversiana extract induced potent antiproliferative effects in all three human colon cancer cells: The antiproliferative activity of the extract against human colon cancer cells (HT-29, HCT-15 and COLO-205) were assessed by MTT assay. Increasing concentrations of the extract $(0,15,25$, $35,45,75$ and $125 \mu \mathrm{g} / \mathrm{mL}$ ) were tested against the cancer cells. All the three colon cancer cells were highly susceptible to the growth inhibitory effect of the extract, but COLO-205 cells were the most affected cancer cells among the three. All the cancer cells showed concentration dependent growth inhibition. Figure 1 shows the effect of the various doses of the extract on the growth inhibition of various colon cancer cells. The $\mathrm{IC}_{50}$ values of the extract against HT-29, HCT-15 and COLO205 cancer cells were found to be 52.1, 43.2 and $38.6 \mu \mathrm{g} /$ $\mathrm{mL}$ respectively indicating that the extract was most active against COLO-205 cells.

Morphological changes induced by A. sieversiana extract in COLO-205 colon cancer cells detected by phase contrast microscopy: Since COLO-205 cancer cells were the most affected among the different tested colon cancer cells. We performed further experiments on COLO-205 cells only. Morphological study using phase contrast microscopy revealed that the extract induced growth inhibition and cellular shrinkage in COLO-205 colon cancer cells. As shown in Figure 2 A-D, the morphological changes indicate that the cells in the extract-treated and the control groups differ substantially. Some of the cells removed from the surface and debris were also detected in the plate of the $A$. sieversiana extract-treated group, but the control (untreated cells) cells were well spread with flattened morphology. The number of cells with shrinkage increased with increase in extract concentration.

Morphological changes in COLO-205 colon cancer cells detected by fluorescence microscopy using acridine orange with ethidium bromide: In case of fluorescence microscopy, COLO-205 cells were stained and evaluated for nuclear shape using a fluorescence microscope with acridine orange with ethidium bromide staining (Figure 3A-D). Acridine orange permeates throughout the cells and renders the nuclei green. Ethidium bromide is taken up by the cells only when cytoplasmic membrane loses its integrity, and stains the nuclei red. Normal cells appear as green while as apoptotic cells appear as red fluorescence. It can be easily seen from the Figure 3 that the extract induced potent apoptotic cascade in the COLO-205 cells in a concentration dependent manner. As compared to the control cells (Figure 3A), A. sieversiana extract-treated cells showed significant fraction of cells showing red fluorescence (Figure 3B-D).

Mitochondrial membrane potential (MMP) loss induced by A. sieversiana extract in COLO-205 cells: Rhodamine-123 is the mostly used dye for determining mitochondrial membrane potential (MMP) loss in cells. In this study,

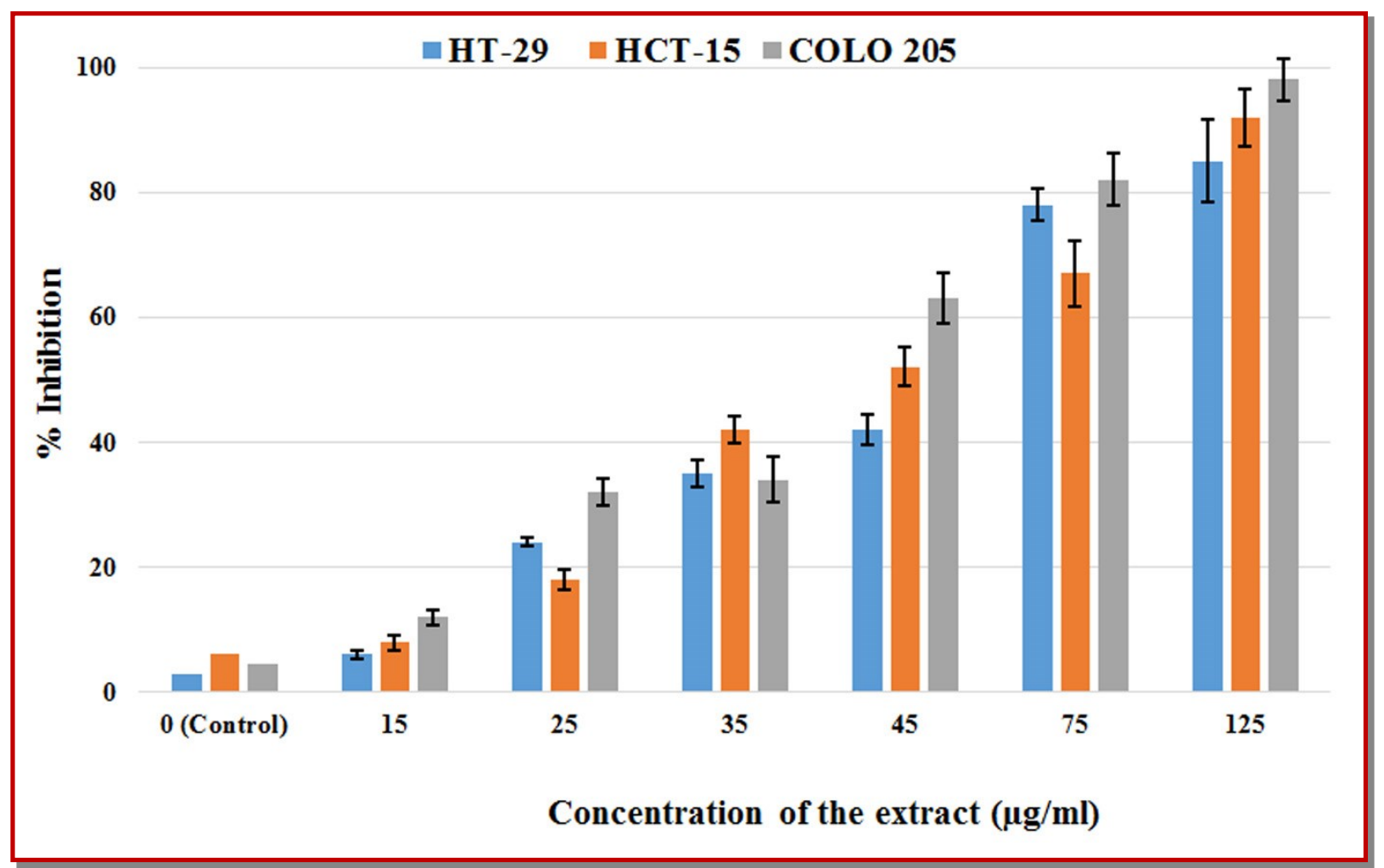

Figure 1: Growth inhibitory effect of ethanol extract of Artemisia sieversiana in three human colon cancer cells (HT-29, HCT-15, COLO-205) at different extract doses after 48 hours. Data are expressed as the mean \pm SD of three independent experiments 


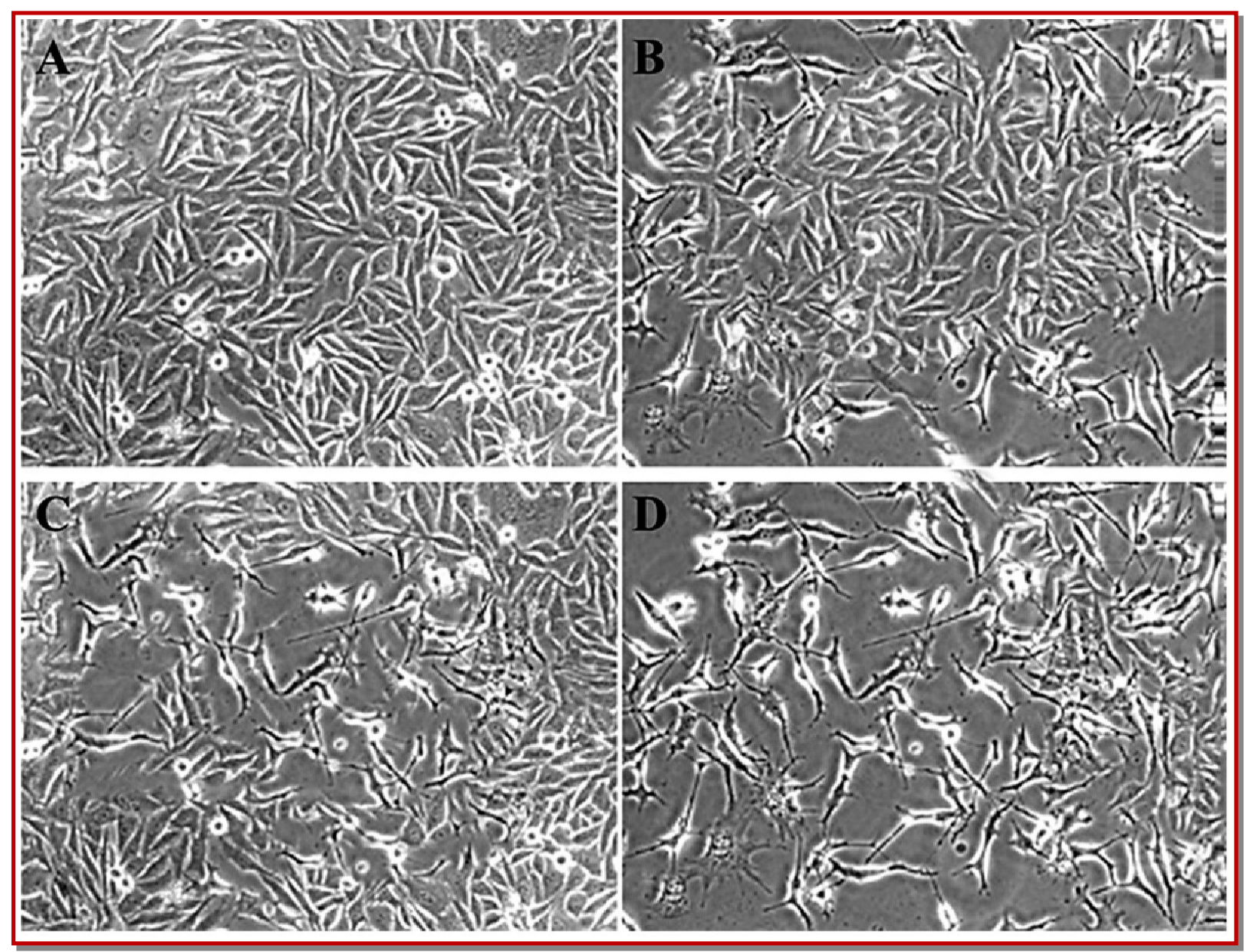

Figure 2: Phase contrast microscopic investigation of COLO-205 cellular morphology following A. sieversiana extract treatment at different doses. A represents untreated cells, B, C and D represent cells treated with 25,45 and $75 \mu \mathrm{g} / \mathrm{mL}$ of the extract

we evaluated the effect of $A$. sieversiana extract on MMP loss in COLO-205 colon cancer cells using flow cytometry. The results are shown in Figure 4, which indicates that the extract induced a dose-dependent loss of MMP in COLO-205 cancer cells. The number of cells with intact mitochondrial membrane potential reduced noticeably while as the number of cells with decreased mitochondrial membrane potential increased. Three different concentrations of the extract $(25,45$ and $75 \mu \mathrm{g} /$ $\mathrm{mL}$ ) were used in the experiment taking untreated cells as the control.

A. sieversiana extract induced potent DNA damage in COLO-205 cells: DNA fragmentation along with the chromatin condensation is the hallmarks of cellular apoptosis process. In the current study, DNA fragmentation of $A$. sieversiana extract-treated cells was studied by observation of the formation of DNA ladder. As shown in Figure 5, DNA ladder appeared to be more marked with the increasing extract dose, however, no DNA fragments were observed in the control groups (Figure 5, left panel). This effect was seen to increase with the increasing dose of the extract.

\section{Discussion}

Previous phytochemical investigations on A. sieversiana have revealed that flavonoids, lignans, terpenoids, sesquiterpene lactones, guaianolides etc are the main chemical constituents of this plant (Bohlmann et al., 1985; Tan et al., 1998; Tang et al., 2000; Suleimenov et al., 2007). Various extracts of $A$. sieversiana have been found to exert strong insecticidal activity against Callosobruchus chinensis, maize weevil Sitophilus zeamais Motsch. A. sieversiana also contains essential oil in its aerial parts and various volatile chemical components have been identified by gas chromatography mass spectrometry. These components include eucalyptol $(9.2 \%)$, geranyl butyrate $(9.1 \%)$, borneol $(7.9 \%)$, camphor $(7.9 \%)$, germacrene D $(5.5 \%)$ and caryophyllene (5.3\%) (Yuan et al., 2007; Liu et al., 2010).

In the present study, we report the anticancer activity of the ethanol extract of $A$. sieversiana in three colon cancer cell lines (HT-29, HCT-15 and COLO-205) along with reporting its effect on apoptosis induction, mitochondrial membrane potential and DNA damage in COLO- 

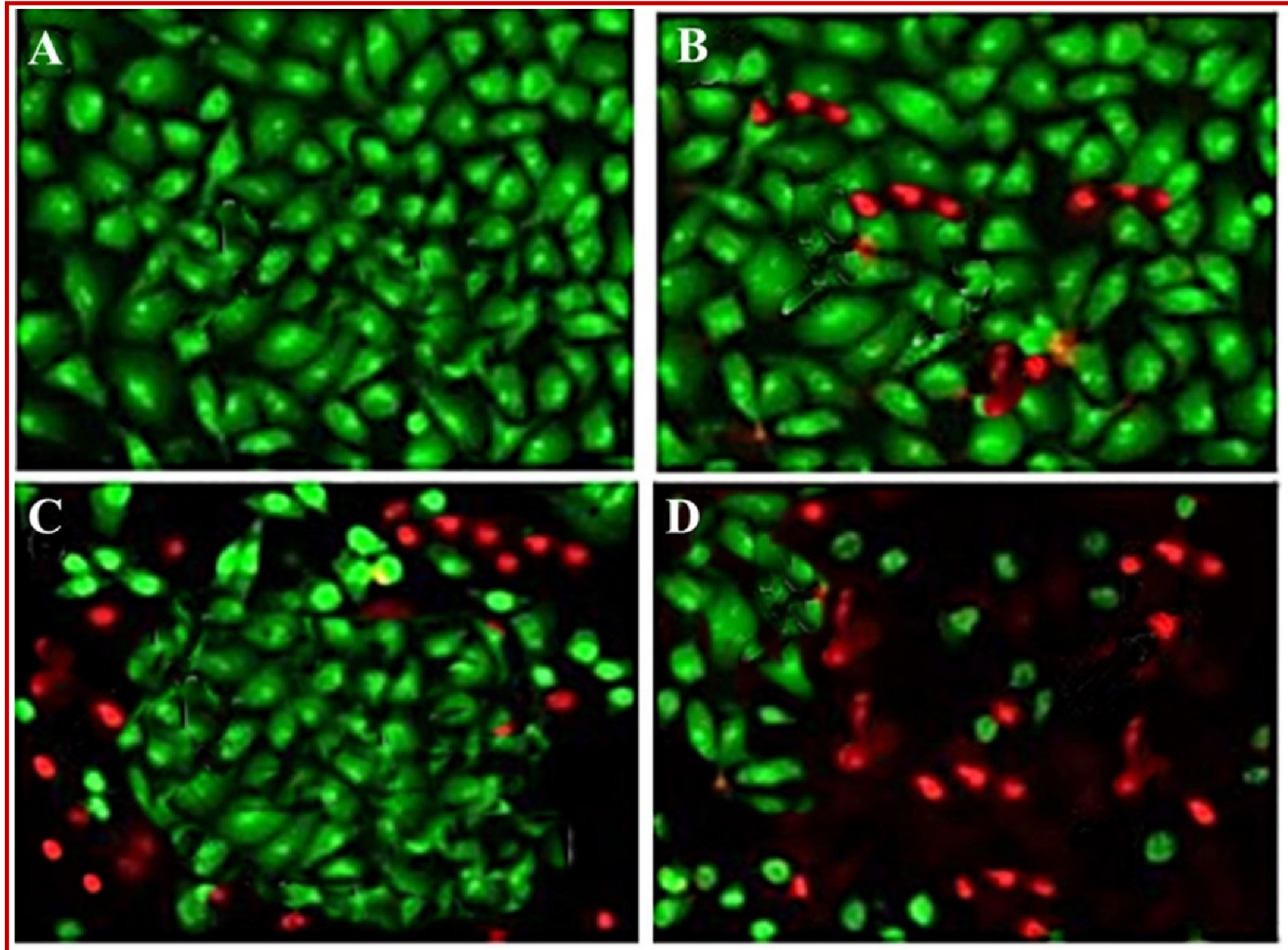

Figure 3: Fluorescence microscopic investigation of COLO-205 cellular morphology following A. sieversiana extract treatment at different doses. A represents untreated cells, B, C and D represent cells treated with 25,45 and $75 \mu \mathrm{g} / \mathrm{mL}$ of the extract

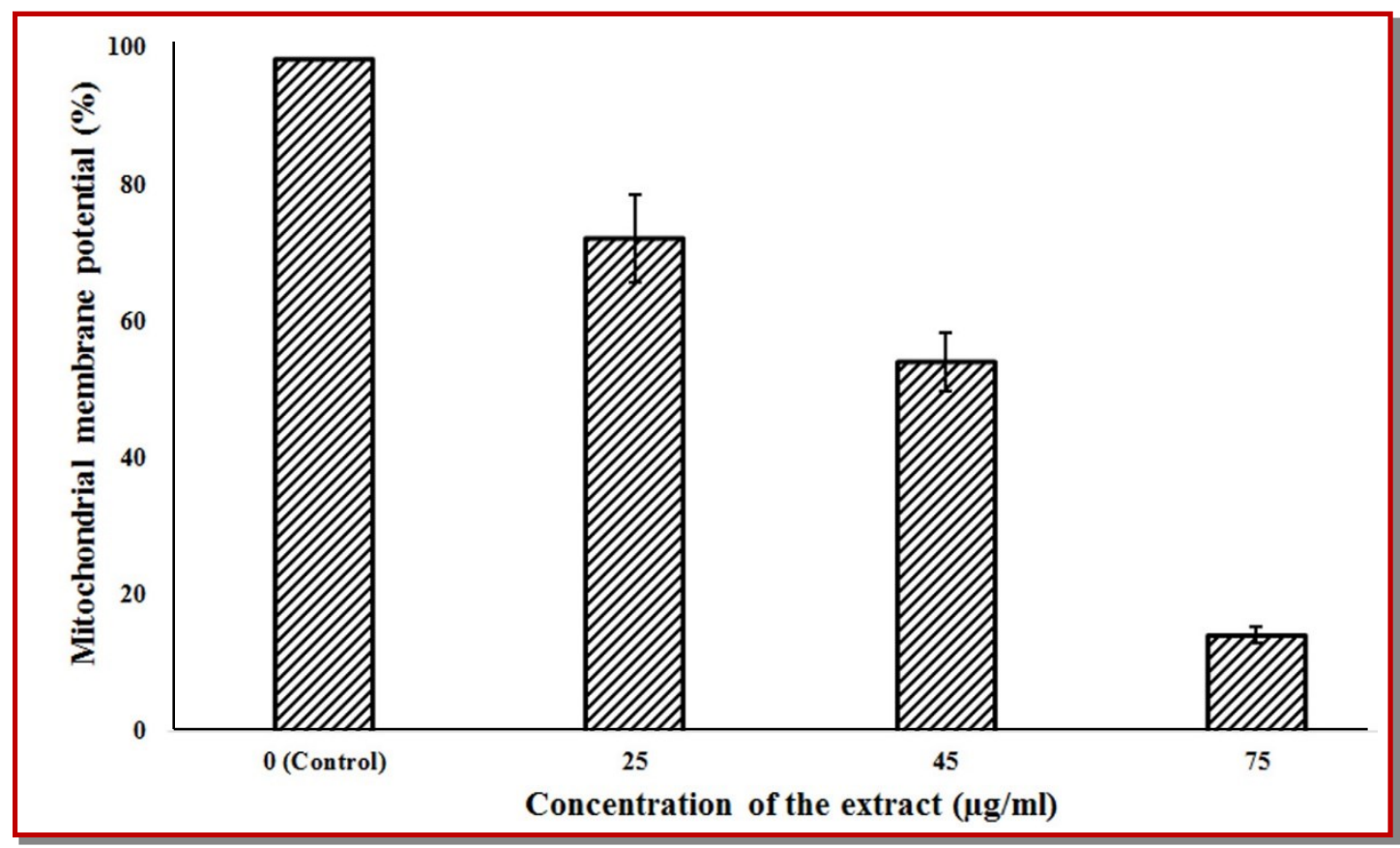

Figure 4: Effect of different doses of the herbal extract of $A$. sieversiana on the mitochondrial membrane potential $(\Delta \Psi \mathrm{m})$ in COLO-205 cancer cells. There is a dose-dependent loss of $\Delta \Psi \mathrm{m}$ in these cells following treatment with increasing doses of the extract 


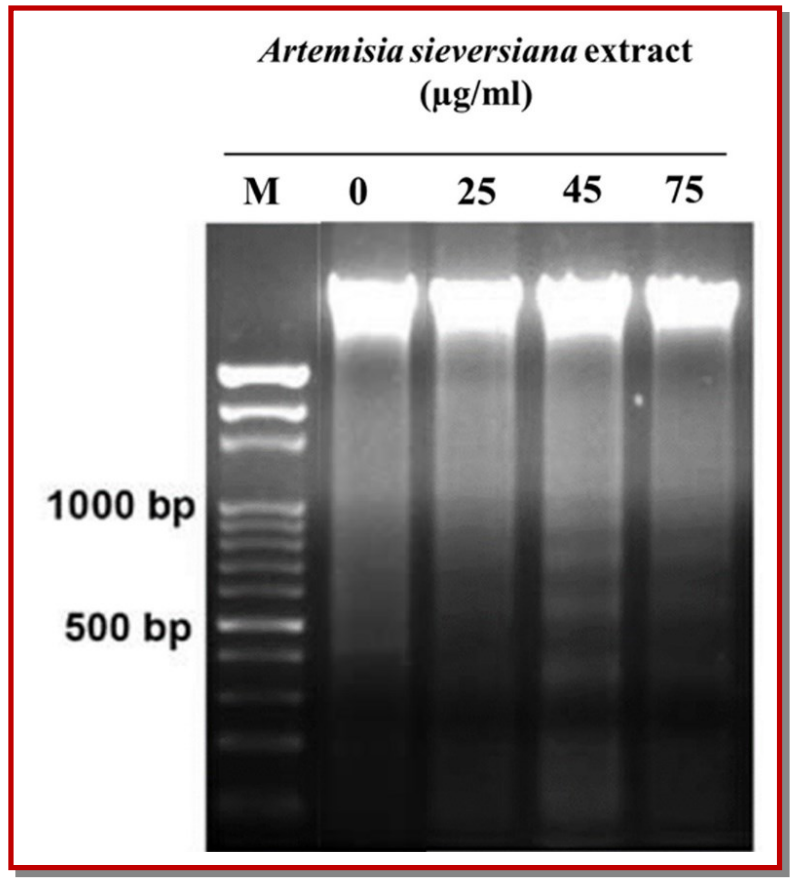

Figure 5: Effect of the herbal extract of A. sieversiana on the DNA fragmentation in COLO-205 cancer cells. Three different concentrations viz., 25,45 and $75 \mu \mathrm{g} / \mathrm{mL}$ were used in the experiment. DNA ladder appeared to be more marked with the increasing extract dose, however, no DNA fragments were observed in the control group

205 cells. To the best of our knowledge, this report constitutes the first such report on this plant species. The results of this investigation reveal that ethanol extract of $A$. sieversiana induces a potent cytotoxic effect on all three colon cancer cell lines with COLO-205 cell line being the most affected one. Further, using phase contrast and fluorescence microscopy, we also investigated the effect of the extract on cellular morphology. The extract induced a significant distortion on the cellular morphology of COLO-205 cancer cells. Loss of mitochondrial membrane potential was observed by flow cytometry following extract treatment at increasing doses. DNA ladder appeared to be more marked with the increasing extract dose, however, no DNA fragments were observed in the control groups.

In conclusion, herbal extract of $A$. sieversiana exhibits anticancer effects in COLO-205 cells through the induction of apoptosis, loss of mitochondrial membrane potential and DNA damage.

\section{Conflict of Interest}

The authors declare that there is no conflict of interest to disclose.

\section{References}

Bohlmann F, Ang W, Trinks C, Jakupovic J, Huneck S. Dimeric guaianolides from Artemisiasieversiana. Phytochemistry 1985; 24, 1009-15.

Jemal A, Bray F, Center MM, Ferlay J, Ward E, Forman D. Global cancer statistics. CA Cancer J Clin. 2011; 61: 69-90.

Jiangsu College of New Medicine, A comprehensive dictionary of the traditional Chinese medicines, Suzhou Jiangsu, China, 1977, pp 627-29.

Kinzler KW, Vogelstein B. Vogelstein: Lessons from hereditary colorectal cancer. Cell 1996; 87: 159-70.

Liu ZL, Liu QR, Chu SS, Jiang GH. Insecticidal activity and chemical composition of the essential oils of Artemisia lavandulaefolia and Artemisia sieversiana from China. Chem Biodivers. 2010; 7: 2040-45.

Mano MS, Duhoux F. Colon cancer: Update on adjuvant therapy. Clin Colorectal Cancer. 2008; 7: 178-83.

Martin D, Leonardo M. Microscopic quantitation of apoptotic index and cell viability using vital and fluorescent dyes. In: Current protocols in immunology. Coligan JE, Kruisbeek AM, Margulies D, Shevach EM, Strober W (eds). New York, John Wiley \& Sons, 1994.

Moertel CG. Chemotherapy for colorectal cancer. N Engl J Med. 1994; 330: 1136-42.

O'Connor ES, Greenblatt DY, LoConte NK, Gangnon RE, Liou JI, Heise CP, Smith MA. Adjuvant chemotherapy for stage II colon cancer with poor prognostic features. J Clin Oncol. 2011; 29: 3381-88.

Rupnarain C, Dlamini Z, Naicker S, Bhoola K. Colon cancer: Genomics and apoptotic events. Biol Chem. 2004; 385: 44964.

Suleimenov EM, Smagulova FM, Seidakhmetova RB, Aksartov RM, Raldugin VA, Adekenov SM. 4-Epiashantin from Artemisia sieversiana. Chem Nat Compd. 2007; 43: 232-33.

Tan RX, Tang HQ, Hu J, Shuai B. Lignans and sesquiterpene lactones from Artemisia sieversiana and Inula racemosa. Phytochemistry 1998; 49: 157-61.

Tang HQ, Hu J, Yang L, Tan RX. Terpenoids and flavonoids from Artemisia species. Planta Med. 2000; 66: 391-93.

Yuan HB, Shang LN, Zhao DX, Ren BZ. Insecticidal activity of extracts from four species of Artemisia against Callosobruchus chinensis. J Jilin Agric Univ. 2007; 29: 612-15.

Author Info

I Zhi-Hong Li (Principal contact)

e-mail: viewcatli@gmail.com; Tel \& Fax:: 0086-711-3251831

I First two authors contributed equally

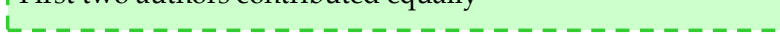

